\title{
Negative refraction in photonic crystals
}

\author{
T. Baba ${ }^{1,2}$ a, T. Matsumoto ${ }^{1,2}$ and T. Asatsuma ${ }^{1,2}$ \\ ${ }^{1}$ Dept. Electrical \& Computer Eng., Yokohama National University, \\ 79-5 Tokiwadai, Hodogayaku, Yokohama 240-8501, Japan \\ ${ }^{2}$ CREST, JST, 5 Samban-cho, Chiyoda-ku, Tokyo 102-0075, Japan \\ a baba@ynu.ac.jp
}

Keywords: Photonic crystal, metamaterials, negative refraction, superlens

\begin{abstract}
Light focusing characteristics of a negative refractive lens fabricated out of a silicon-on-insulator photonic crystal (PC) slab are investigated theoretically and experimentally. It focuses in the near infrared, but the focal spot is degraded by a lens aberration. To reduce the aberration, we designed a composite PC that gives rise to a narrower focal spot. In addition, two unique functions of this lens are demonstrated: refocusing outside of the PC and parallel focusing, enabling image transfer and real image formation, respectively. These results prove the feasibility of an in-plane free space optical network based on negative refraction.
\end{abstract}

\section{Introduction}

Left-handed media with negative refractive indices result in light focusing characteristics that are different from those of conventional positive refractive optical components [1]. Such media are realized by metamaterials composed of metal/dielectric composites. Light propagation in metamaterials, particularly in the near-field regime, has been theoretically analyzed and experimentally observed using millimeter waves and lightwaves [2-5]. Recently it has been shown that negative refraction is also achievable in the far-field regime [6,7]. However, the absorption loss in the metal limits potential optical applications. Photonic crystals (PCs) composed solely of dielectric composites exhibit negative refraction based not on left-handed media but instead on anomalous dispersion arising from the multi-dimensional periodicity. A PC can even focus diverging light owing to the negative refraction at the flat boundary [8]. With regard to such lenses, some theoretical studies predict an image transfer from one side of the PC to the other. Additional predictions include real image formation inside the PC independent of the incident object's position, and subwavelength imaging [9-10]. Initial experiments were carried out using millimeter waves [11-13], while those using lightwaves have been limited [14].

For an effective demonstration of a PC negative refractive lens at optical frequencies, the following three issues must be resolved: 1) nano-scale fabrication process, 2) strong optical confinement in the plane of propagation, and 3) suppression of unwanted reflection and diffraction at the input and output (I/O) ends of the PC. In our previous 
study, we used a PC slab on a silicon-on-insulator (SOI) substrate. It is easily fabricated by forming airholes in the thin, top Si layer using dry etching, and it strongly confines light in the Si layer by total internal reflection at the high-index-contrast boundaries. We optimized the interface between the PC and Si slabs using finite-difference time-domain (FDTD) simulations, so that the reflection and diffraction loss is suppressed to $0.6 \mathrm{~dB}$ [15]. Consequently light focusing was observed inside the PC at near-infrared wavelengths [16]. However, the focal spot was twice as wide as that expected from the mode size of the light source, primarily due to an aberration arising from a slight distortion of the dispersion contours from ideal parabolic ones. In the first part of this paper, we demonstrate a reduction in aberration using a composite PC.

In the second part of this paper, we demonstrate refocusing outside of the PC and parallel focusing as simple examples of image transfer and of real image formation, respectively. Some experiments on refocusing have been already reported $[17,18]$. However, the manner in which light is incident onto and guided in the PC were not clearly evaluated. Therefore, the results may have been influenced by an aperture effect in which the angle of incidence is limited by reflection and diffraction losses and a pseudo-focal spot is formed outside of the PC [19]. In this study, we quantify the light propagation and confirm detailed agreement between the theoretical and experimental results. We also show that multiple light sources are focused at symmetrical positions.

\section{Light focusing in a single PC slab}

Figure 1(a) shows the PC slab (called PC-A) consisting of circular airholes in a square lattice rotated by $45^{\circ}$. We have already reported that the dispersion surface in this structure will focus light at a small incident angle $[15,16]$. The normalized airhole diameter $2 r_{\mathrm{A}} / a_{\mathrm{A}}$ is fixed at 0.624 , where $2 r_{\mathrm{A}}$ is the diameter and $a_{\mathrm{A}}$ is the lattice constant. Figures 1(b) and (c) respectively show equi-frequency contours (i.e., the dispersion surface) of the second band for in-plane polarization and a ray tracing obtained from the gradient of the contour curves. To approximate the PC slab in a two-dimensional (2D) model, we take the equivalent modal index of the slab to be 2.963. To perform the ray tracing, a point source was located $106.4 a_{\mathrm{A}}$ away from the input end of the PC. The incident angle of light $\theta_{\text {in }}$ was limited to $\pm 7^{\circ}$. because low reflection and diffraction losses are maintained for this angular range [15]. As suggested by the inwardly curving contourbs, negative refraction occurs over a large frequency bandwidth. For a normalized frequency of $a_{\mathrm{A}} / \lambda=0.30$, where $\lambda$ is the wavelength, the spot width $2 w$ is $4.0 a_{\mathrm{A}}(=1.2 \lambda)$ at the focal point, defined as the location of the minimum width between rays. Figure 1(d) shows the $\left|H_{\mathrm{z}}\right|^{2}$ distribution (where $H_{\mathrm{z}}$ is the magnetic field component normal to the 2D slab) of the propagating light along with its spatial profile calculated by the FDTD simulation. A light source was located at the same position as in the ray tracing, and a continuous wave was excited with a Gaussian profile having a full width at half maximum (FWHM) of $2.2 a_{\mathrm{A}}$. The optimized interface between the PC and the outer slab 
consists of one row of airholes, which has a fourth order functional shape and triangular shape on the front and back sides, respectively [15]. This interface acts a phase transformer, changing diverging incident light into Bloch waves in the PC. Light is negatively refracted at the interface and focused in the PC. Here, we define the focal position as that of minimum FWHM of the light beam in the calculation. The profile oscillates because of the weak light intensity in the airholes. The spot width $2 w$ evaluated from the FWHM of the envelope profile was $3.9 a_{\mathrm{A}}$, in agreement with the ray tracing. In principle, the negative refractive lens should form an equi-magnification image of the light source in the PC. Therefore $2 w$ should equal $2.2 a_{\mathrm{A}}$ in the ideal case, and the larger value found in the simulation is caused by an aberration.

The fabrication began with a silicon-on-insulator (SOI) substrate having a 0.26 - $\mu \mathrm{m}$-thick $\mathrm{Si}$ layer and a $1-\mu \mathrm{m}$-thick $\mathrm{SiO}_{2}$ layer. We formed the airhole pattern of the $\mathrm{PC}$ slab, as well as the photonic wire waveguide, using $e$-beam lithography and $\mathrm{SF}_{6}$ inductively coupled plasma etching. Finally we formed the air-bridge structure using HF wet etching. Figure 1(e) shows a scanning electron microscope (SEM) image of the device. The lattice constant $a_{\mathrm{A}}$ was $0.47 \mu \mathrm{m}$ and the airhole diameter $2 r_{\mathrm{A}}$ was $0.29 \mu \mathrm{m}$ so
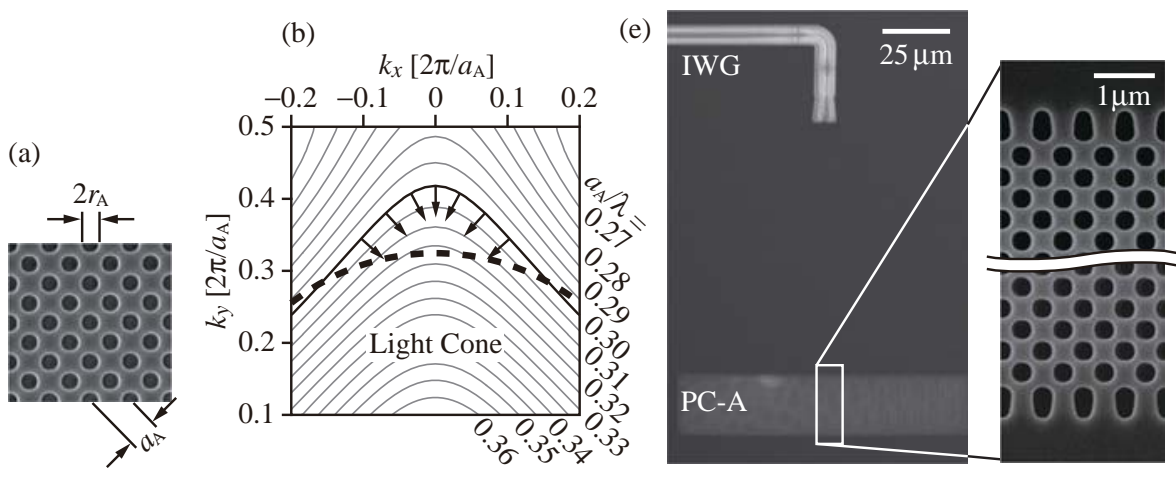

(c)
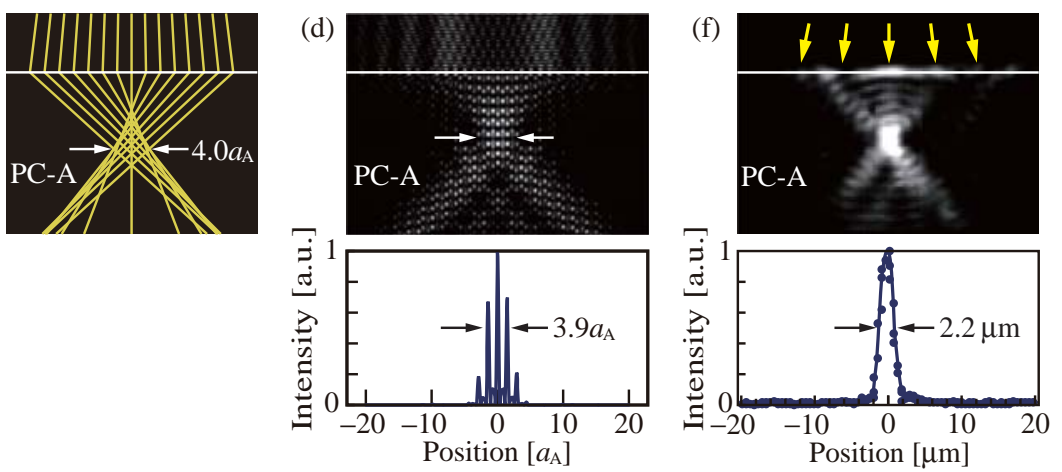

Fig. 1 Structure and theoretical and experimental characteristics of PC-A. (a) Top view of PC-A. (b) Dispersion surface calculated by the two-dimensional plane-wave expansion method. The dashed line and the arrows show the light line and the directions of the Poynting vector, respectively. (c) Ray tracing for $a_{\mathrm{A}} / \lambda=0.30$. (d) Light propagation and focal spot profile calculated by the FDTD method for $a_{\mathrm{A}} / \lambda=0.30$. (e) SEM image of the device, where IWG is the input waveguide. (f) Streak image of the propagating light and focal spot profile at $\lambda=1.355 \mu \mathrm{m}$. 
that $2 r_{\mathrm{A}} / a_{\mathrm{A}}=0.62$. We placed the optimized interface at the $\mathrm{I} / \mathrm{O}$ ends of the PC. To perform the measurements, we coupled tunable laser light into the cleaved facet of the photonic wire waveguide using a 50× objective lens. This waveguide was $2 \mu \mathrm{m}$ in width near the cleaved facet, and tapered to a single mode width of $0.3 \mu \mathrm{m}$. The wet etching time was controlled so that the $\mathrm{SiO}_{2}$ layer beneath the wide part of the waveguide remained. For the narrow part, we arranged cross beams having an elliptical intersection in the waveguide, to mechanically support the air-bridge waveguide formed after the wet etching while minimizing transmission losses [20]. The photonic wire waveguide was connected to the Si slab through a parabolic taper of $2.3 \mu \mathrm{m}$ width that expanded the guided light to a modal FWHM of $1.1 \mu \mathrm{m}\left(2.3 a_{\mathrm{A}}\right)$. Subsequently, it was further expanded in the Si slab of $100 \mu \mathrm{m}\left(212.8 a_{\mathrm{A}}\right)$ length as a Gaussian beam, and was incident on the PC. (This length was employed for clear observation of the focusing.) We observed the light propagation from above the slab using an InGaAs image sensor through a $100 \times(\mathrm{NA}=$ 0.5 ) objective lens. Figure 1(f) shows a streak image of the light propagation in the PC and the light intensity profile at the focal spot for $\lambda=1.355 \mu \mathrm{m}\left(a_{\mathrm{A}} / \lambda=0.35\right)$. This wavelength is inside the light cone, and therefore the light in the PC is not perfectly guided but is instead partly radiated out of the slab. In consequence, the propagation could be directly observed. Light focusing based on negative refraction was confirmed, with a focal length of $\sim 5 \mu \mathrm{m}$. In this process, light did not reach the output end of the PC due to radiation losses. But at longer wavelengths outside the light cone, the streak image in the PC disappeared while light scattering at the output end of the PC became visible. This indicates that light reached the output end with minimal losses. Returning to the streak image in Fig. 1(f), we see that the light appears to be divided into two branches beyond the focal spot, because only the equi-frequency contour of the center light is located outside of the light cone, as shown in Fig. 1(b). Here the spot width $2 w$ was 2.2 $\mu \mathrm{m}\left(4.7 a_{\mathrm{A}}\right)$, slightly wider than in the simulation. Two factors could affect the focal spot: radiation losses not included in the $2 \mathrm{D}$ simulation, and fabrication errors in the optimized interface resulting in a narrower accepted angle of light.

\section{Composite PC lens for aberration reduction}

We propose a composite PC, in which PC-A is cascaded to a second slab called PC-B, which has opposite aberration characteristics so that the net aberration is zero. As understood from Figs. 1(b) and (c), the focal length increases with the incident angle in PC-A. Therefore a shortening of the focal length at wider angles is needed for PC-B. Numerical exploration led to a structure consisting of elliptical airholes in a triangular lattice, as shown in Fig. 2(a). The normalized airhole diameters in the vertical and lateral directions, $2 r_{\mathrm{BV}} / a_{\mathrm{B}}$ and $2 r_{\mathrm{BL}} / a_{\mathrm{B}}$, were optimized as 1.08 and 0.54 , respectively, where $a_{\mathrm{B}}$ is the lattice constant of PC-B. For the smooth propagation of Bloch waves across the boundary between the two PCs, the pitch of the airholes on the boundary should be the same, i.e., $\sqrt{2} a_{\mathrm{A}}=a_{\mathrm{B}}$. The thick solid lines in Figs. 1(b) and 2(b) show the equi-frequency 
(b)

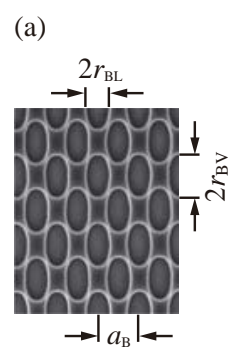

$k_{x}\left[2 \pi / a_{\mathrm{B}}\right]$

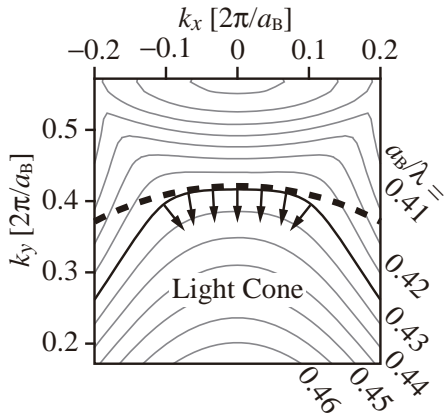

(e)

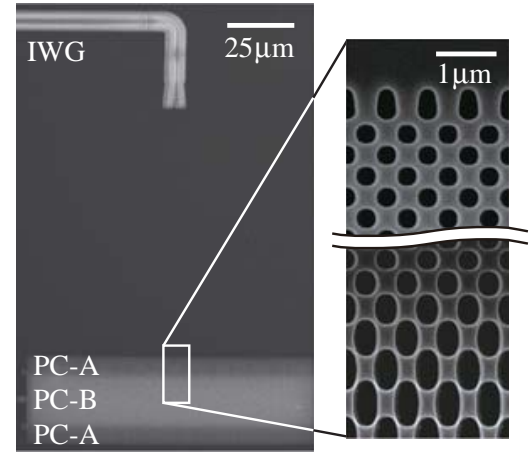

(c)
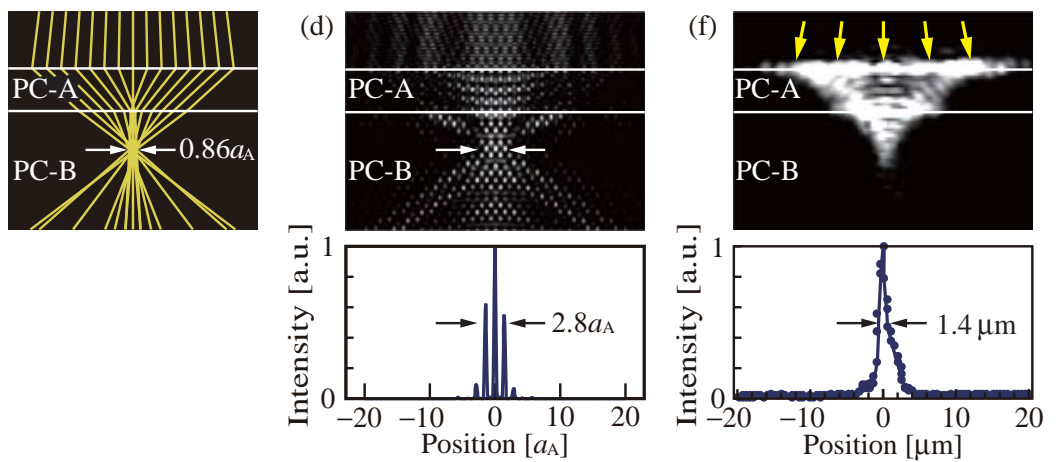

Fig. 2 Structure and theoretical and experimental characteristics of the composite PC. (a) Top view of PC-B. (b) Dispersion surface calculated by the two-dimensional plane-wave expansion method. The dashed line and the arrows show the light line and the directions of the Poynting vector, respectively. (c) Ray tracing for $a_{\mathrm{A}} / \lambda=0.30$. (d) Light propagation and focal spot profile calculated by the FDTD method for $a_{\mathrm{A}} / \lambda=0.30$. (e) SEM image of the device. (f) Streak image of the propagating light and focal spot profile at $\lambda=1.325 \mu \mathrm{m}$.

contours in PC-A and PC-B at $a_{\mathrm{A}} / \lambda=0.30$ and $a_{\mathrm{B}} / \lambda=0.43$, respectively. These contours lie close to the boundary of the light cone shown by the dashed line. At lower frequencies, both PCs satisfy the lossless condition outside of the light cone. At slightly higher frequencies, the contour of PC-B enters the light cone and the light propagation can be observed from above. Figure 2(c) shows the ray tracing in the composite PC. The parameters are the same as those for Fig. 1(c). The length of PC-A was optimized to be $5.6 a_{\mathrm{A}}$ so as to minimize the aberration. The spot width $2 w$ in this simulation was $0.86 a_{\mathrm{A}}=$ $0.26 \lambda$, which is 4.7 times smaller than for the single PC and is smaller than the diffraction limit, demonstrating suppression of the aberration in the composite PC. To confirm this, we performed a FDTD simulation for this structure with the same calculation parameters as used for Fig. 1(d). The results are shown in Fig. 2(d). In a preliminary calculation, a large reflection and diffraction loss of $7.8 \mathrm{~dB}$ was observed at the boundary when PC-A and PC-B were directly connected. The loss was reduced to $3.5 \mathrm{~dB}$ when the airhole diameter and the vertical lattice constant of the two rows of airholes near the boundary were adiabatically changed. It still has room for improvement. But we already observe 
light transmission and tight focusing in PC-B. Here, $2 w$ evaluated from the envelope profile is $2.8 a_{\mathrm{A}}$. Considering the limitation of the light source's width, this result is estimated to contain only a third of the aberration of the single PC. The small value of $2 w$ and the focal spot intensity are maintained over the $4 \%$ bandwidth of the center frequency (more than $50 \mathrm{~nm}$ at $\lambda=1.5 \mu \mathrm{m})$. It covers the C-band $(\lambda=1.530-1.565 \mu \mathrm{m}$ ) of silica fiber communications.

Figure 2(e) shows a SEM picture of the fabricated device. PC-A is the same as that in Section 2. In PC-B, $a_{\mathrm{B}}=0.665 \mu \mathrm{m}, 2 r_{\mathrm{BV}}=0.72 \mu \mathrm{m}\left(2 r_{\mathrm{BV}} / a_{\mathrm{B}}=1.08\right)$, and $2 r_{\mathrm{BL}}=0.37 \mu \mathrm{m}$ $\left(2 r_{\mathrm{BL}} / a_{\mathrm{B}}=0.56\right)$. In the experiment, the Si slab was fixed at $100 \mu \mathrm{m}$ in length, which was twice that in the FDTD simulation. Accordingly, the number of rows of airholes in the adiabatic structure was set as four. Figure 2(f) shows the light propagation and the intensity profile of the focal spot at $\lambda=1.325 \mu \mathrm{m}\left(a_{\mathrm{B}} / \lambda=0.50\right)$. The focal spot narrowed as compared with that for the single PC. Light rapidly decayed beyond the spot, possibly due to the stronger radiation of the light cone in PC-B which has large airholes. The spot width $2 w$ was $1.4 \mu \mathrm{m}\left(3.0 a_{\mathrm{A}}\right)$, which again is slightly wider than the theoretical result. Figure 3 compares the wavelength dependence of $2 w$ for the single and composite PC. The latter exhibits a narrower $2 w$ over the entire wavelength range of the measurement. We conclude that the aberration is reduced in the composite PC. In general, the focal spot in the negative refractive lens should be narrower as the light source becomes narrower. However, once the divergence of the incident light equals the acceptance angle $\left( \pm 7^{\circ}\right.$ in the present case), narrowing the width of the light source does not decrease the focal size any further. For the further reduction of the focal spot, it is essentially important to expand the acceptance angle, together with the reduction in aberration.

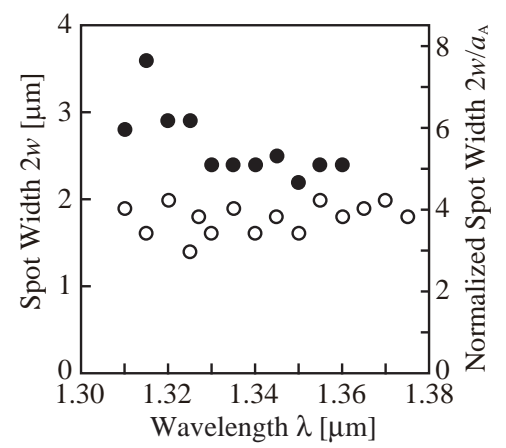

Fig. 3 Measured wavelength dependence of the focal spot width $2 w$ for PC-A (closed circles) and for the composite PC (open circles).

\section{Light focusing outside of the PC and parallel focusing}

When the PC has parallel I/O ends, light diverging beyond the focal point in the PC is refocused outside of the PC. In other words, the image of the light source is transferred to 
the far side of the PC. As a demonstration, we characterized the coupling of light from an input waveguide to output waveguides. Figure 4(a) shows the fabricated device. The length of the slab between the input waveguide and the PC (PC-A in this experiment) was $50 \mu \mathrm{m}$. The length of the PC was twice as long as the focal length, i.e., $10.6 \mu \mathrm{m}$ for $\lambda \sim$ $1.5 \mu \mathrm{m}$, so that the focal point was located at the center of the PC. The output interface was symmetric to the input interface, with nine output waveguides arranged in parallel. A parabolic taper was arranged at the end of each waveguide. The light at the other end of the output waveguides was used to evaluate the refocusing. As illustrated in Fig. 4(b), focusing was observed at $\lambda \leq 1.34 \mu \mathrm{m}$. Because the radiation loss was suppressed, light is focused inside the PC, refocused outside the PC, and then extracted from the center output waveguide. The same refocusing occurs at $\lambda>1.34 \mu \mathrm{m}$, although the streak image disappeared, and a strong output was observed at $\lambda=1.48 \mu \mathrm{m}$. This output disappeared at $\lambda=1.55 \mu \mathrm{m}$. Figure 5(a) compares the measured transmission spectrum (blue line) for the center output waveguide with the theoretical curve (black line) from the FDTD simulation. The gray region is the light cone, which was not included in the 2D simulation. Therefore, the rapid drop within the light cone does not appear in the simulation. The drop at longer wavelengths is due to chromatic aberration; the focal length changes significantly with wavelength. Figure 5(b) shows a ray tracing and FDTD simulation at $\lambda$ $=1.48 \mu \mathrm{m}$, for which the output intensity is a maximum. The I/O waveguides were not considered in the ray tracing, but they were included in the FDTD simulation. The calculated refocusing supports the experimental results. In the ray tracing, the focal point is located beyond the end of the output waveguide. The beam profile becomes multimodal and the coupling efficiency is degraded at the shifted position.
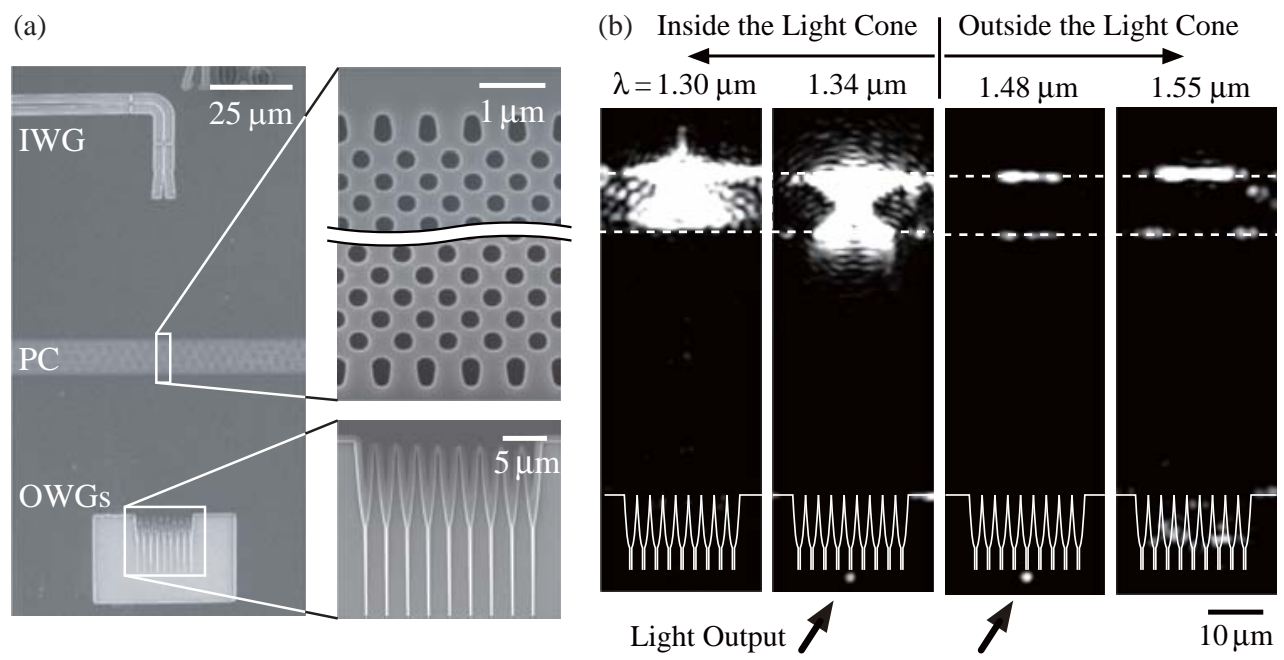

Fig. 4 SEM image and streak images of light refocusing outside of the PC. (a) Top view of the fabricated device, where OWGs are the output waveguides. (b) Streak images superimposed with white lines denoting the PC and OWGs. The arrows indicate the extracted light. 
(a)

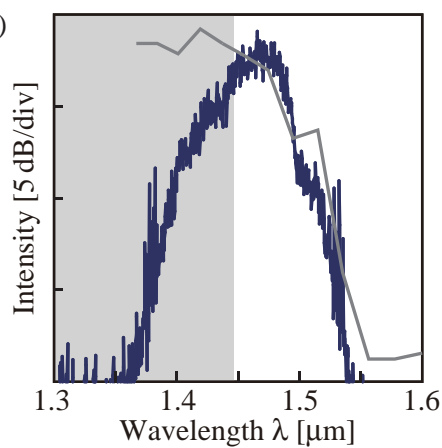

(b)

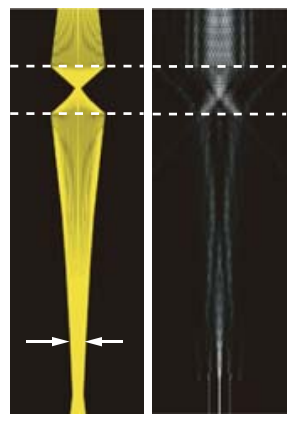

Fig. 5 Experimental and theoretical results of light refocusing outside of PC-A. (a) Transmittance through the center OWG in the experiment (blue line) and in the FDTD simulation (gray line). The gray region indicates the wavelength range inside the light cone. (b) Ray tracing and FDTD simulation at $\lambda=1.48 \mu \mathrm{m}$. In this ray tracing, $\theta_{\text {in }}$ is limited to $\pm 6.6^{\circ}$. The arrows indicate the input end of the center OWG.

(a)

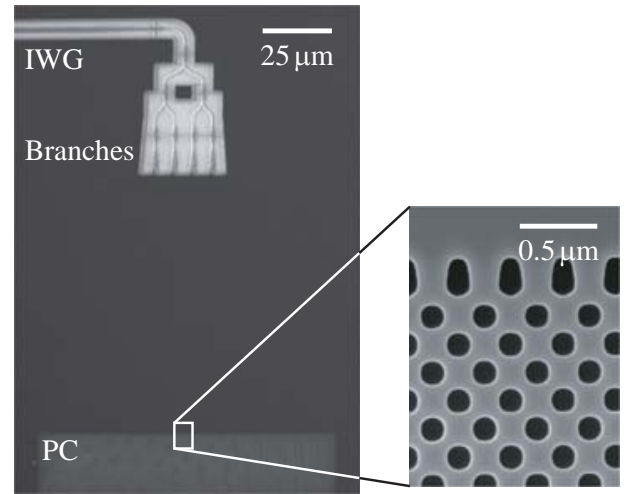

(b)

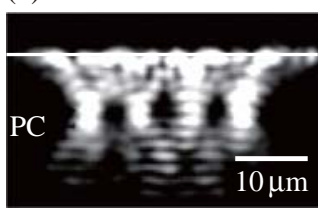

(c)

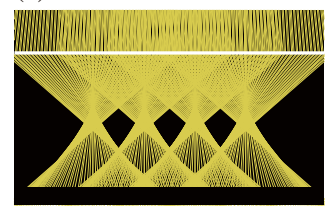

(d)

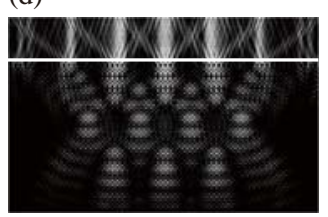

Fig. 6 SEM image of PC-A and demonstration of parallel focusing. (a) Top view of the fabricated device. (b) Streak image of light propagation at $\lambda=1.30 \mu \mathrm{m}$. (c) Ray tracing. (d) FDTD simulation of light propagation.

To demonstrate parallel focusing, we prepared multiple light sources; the input waveguide was divided into four branches through bend-waveguide splitters [21]. The same parabolic taper was arranged at the end of each branch. The pitch of the four branches was $7 \mu \mathrm{m}$, and the slab length was 50 or $100 \mu \mathrm{m}$. Light was incident on the PC (PC-A in this case) with the optimized interface. Figures 6(a) and (b) show SEM pictures of the device and a streak image of the propagating light at $\lambda=1.30 \mu \mathrm{m}$. The image of each light source is formed at a symmetrical position inside the PC. The focal spot widths 
and focal lengths are approximately uniform and constant over the wavelength range from $1.265-1.300 \mu \mathrm{m}$. Ray tracing and FDTD simulations agree with these experimental results, as shown in Figs. 6(c) and (d), respectively. We have used the parallel focusing to construct a compact wavelength demultiplexer consisting of a PC superprism (serving as a diffraction grating) and a PC lens (serving as a condenser lens) [22]. The combination of parallel focusing and refocusing allows a parallel optical coupler between the waveguides and other optical devices.

\section{Summary}

We have experimentally demonstrated some unique light focusing characteristics of a negative refractive lens based on the anomalous dispersion of a photonic crystal. Light focusing in a single PC was observed at near-infrared wavelengths. Compared with previous work, as the device size is enlarged the light propagation is magnified. From ray tracings, FDTD simulations, and experiments, it has been demonstrated that aberration is the major cause of the expansion of the focal spot to $2.2 \mu \mathrm{m}\left(4.7 a_{\mathrm{A}}\right)$. To counteract this effect, a composite PC was fabricated, in which two PCs having opposite aberration characteristics were cascaded. It achieved a narrower focal spot width of $1.4 \mu \mathrm{m}\left(3.0 a_{\mathrm{A}}\right)$, in which the aberration component was suppressed to $0.3 \mu \mathrm{m}$. We also demonstrated refocusing outside of the PC as well as parallel focusing, which are consistent with theoretical results. They have application to a compact demultiplexer and a parallel optical coupler. Furthermore, we expect such negative refractive optics to provide more sophisticated functions for in-plane free space optical networks.

\section{References}

[1] V. G. Veselago: Sov. Phys. Usp. Vol.10 (1968), p.509

[2] J. B. Pendry: Phys. Rev. Lett. Vol.85 (2000), p.3966

[3] R. A. Shelby, D. R. Smith and S. Schultz: Science Vol. 292 (2001), p.77

[4] N. Fang, H. Lee, C. Sun and X. Zhang: Science Vol. 308 (2005), p.534

[5] V. M. Shalaev: Nat. Photonics Vol. 1(2007), p.41

[6] I. Smolyaninov, Y. Hung and C. Davis: Science Vol. 315 (2007), p.1699

[7] Z. Liu, S. Durant, H. Lee, Y. Pikus, Y. Xiong, C. Sun and X. Zhang: Opt. Express Vol. 15(2007), p.6947

[8] H. Kosaka, T. Kawashima, A. Tomita, M. Notomi, T. Tamamura, T. Sato and S. Kawakami: Appl. Phys. Lett. Vol. 74 (1999), p.1212

[9] M. Notomi: Phys. Rev. Vol. B62 (2000), p.10696

[10] C. Luo, S. G. Johnson and J. D. Joannopoulos: Phys. Rev. Vol. B65 (2002), p. 201104 
[11] E. Cubukcu, K. Aydin and E. Ozbay: Phys. Rev. Lett. Vol. 91 (2003), p.207401

[12] P. V. Parimi, W. T. Lu, P. Vodo and S. Sridhar: Nature Vol. 426 (2003), p.404

[13] Z. Lu, C. Chen, C. A. Schuetz, S. Shi, J. A. Murakowski, G. J. Schneider and D. W. Prather: Appl. Phys. Lett. Vol. 87 (2005), p.091907

[14] A. Berrier, M. Mulot, M. Swillo, M. Qiu, L. Thylén, A. Talneau and S. Anand: Phys. Rev. Lett. Vol. 93 (2004), p.073902

[15] T. Matsumoto, S. Fujita and T. Baba: Opt. Express Vol. 13 (2005), p.10768

[16] T. Matsumoto, K. Eom and T. Baba: Opt. Lett. Vol. 31 (2006), p.2776

[17] E. Schonbrun, T. Yamashita, W. Park and C. J. Summers: Phys. Rev. Vol. B73 (2006), p.195117

[18] Z. Lu, B. Miao, T. R. Hodson, C. Lin, J. A. Murakowski and D. W. Prather: Opt. Express Vol. 15(2007), p.1286

[19] T. Matsumoto and T. Baba: Spring Meet. Jpn. Soc. Appl. Phys (2005), 29p-YV-5

[20] T. Fukazawa, T. Hirano, F. Ohno and T. Baba: Jpn. J. Appl. Phys. Vol. 43 (2004), p.646

[21] A. Sakai, T. Fukazawa and T. Baba: IEICE Trans. Electron. (2002), E85-C, p.1033

[22] T. Matsumoto, T. Asatsuma and T. Baba: Appl. Phys. Lett. Vol. 91 (2007), p.091117 